\title{
Cytoreductive Surgery and Hyperthermic Intraperitoneal Chemotherapy in Gastric Cancer with Peritoneal Metastasis-Indian Experience
}

\author{
Somashekhar S.P. ${ }^{1}$ (1) Jyothsana Karivedu ${ }^{1}$ Rohit Kumar C. ${ }^{1}$ Ramya Y. ${ }^{2}$ Priya Kapoor ${ }^{1}$ \\ Amit Rauthan ${ }^{3}$ Ashwin K.R. ${ }^{1}$ \\ ${ }^{1}$ Department of Surgical Oncology, Manipal Comprehensive Cancer \\ Center, Manipal Hospital, Bangalore, Karnataka, India \\ 2 Department of Surgical Oncology, Apollo Hospital, Mysore, \\ Karnataka, India \\ Address for correspondence Dr. Ashwin K.R., DNB, Department of \\ Surgical Oncology, Manipal Comprehensive Cancer Center, Manipal \\ Hospital, Bangalore 560017, Karnataka, India \\ (e-mail: doc.ashwin.kr@gmail.com).
}

${ }^{3}$ Department of Medical Oncology, Manipal Comprehensive Cancer Center, Manipal hospital, Bangalore, Karnataka, India

South Asian J Cancer 2022;11(2):121-124.

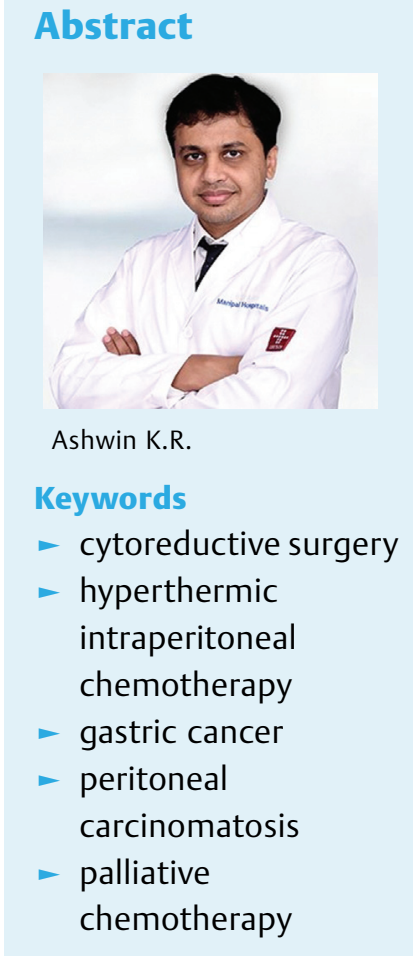

Introduction Peritoneal metastasis secondary to gastric cancer is associated with poor prognosis. Recent studies have shown that cytoreductive surgery (CRS) and hyperthermic intraperitoneal chemotherapy (HIPEC) may be an efficacious treatment option for an otherwise palliative condition.

Methods A retrospective single institutional study of patents diagnosed with gastric carcinoma and peritoneal metastasis and treated with CRS and HIPEC from February 2015 to December 2019.

Results Sixteen patients with gastric cancer and peritoneal carcinomatosis were treated with CRS and HIPEC. Three patients underwent upfront surgery, and five patients underwent interval surgery. The mean peritoneal cancer index $(\mathrm{PCI})$ was 3.5 , and adequate complete cytoreduction (CC) score of $0 / 1$ was achieved in all patients. All patients received HIPEC with mitomycin C. Major surgical complications were in 12.5\% of patients. Grade I surgical site infection was present in one patient. Three patients had prolonged gastrointestinal (GI) recovery. The 30-day mortality was zero. Median follow-up time was 39 months. The median progression-free survival (PFS) was 12 months (95\% confidence interval $[\mathrm{Cl}] 6.86-17.13$ ). The median overall survival (OS) was 17 months (95\% Cl 6.36-27.64).

Conclusion Multidisciplinary treatment of perioperative chemotherapy with CRS and HIPEC is a promising treatment option, which may prolong survival in selected patients, and large randomized clinical trials are warranted for it to become standard of care.

DOI https://doi.org/10.1055/s-0041-1739176 ISSN 2278-330X

How to cite this article: SP S, Karivedu J, C RK, et al. Cytoreductive Surgery and Hyperthermic Intraperitoneal Chemotherapy in Gastric Cancer with Peritoneal Metastasis-Indian Experience South Asian J Cancer 2022;11(2):121-124. (c) 2021. MedIntel Services Pvt Ltd. All rights reserved.

This is an open access article published by Thieme under the terms of the Creative Commons Attribution-NonDerivative-NonCommercial-License, permitting copying and reproduction so long as the original work is given appropriate credit. Contents may not be used for commercial purposes, or adapted, remixed, transformed or built upon. (https://creativecommons.org/licenses/by-nc-nd/ 4.0/)

Thieme Medical and Scientific Publishers Pvt. Ltd., A-12, 2nd Floor, Sector 2, Noida-201301 UP, India 


\section{Introduction}

We analyze the results of the treatment with cytoreductive surgery (CRS) and hyperthermic intraperitoneal chemotherapy (HIPEC) for patients with gastric cancer and peritoneal carcinomatosis in Indian patients.

\section{Methods}

This is as retrospective single institutional study of patents diagnosed with gastric carcinoma and peritoneal metastasis and treated with CRS and HIPEC from February 2015 to December 2019. All the patients with resectable peritoneal disease and no metastatic disease, Eastern Cooperative Oncology Group (ECOG) 0/1, $>70$ years of age, and preoperative serum albumin $>3 \mathrm{~g} \%$ were included in the study ( - Table 1). To be considered for CRS and HIPEC, patients should have a normal organ function (serum creatinine $<1.5 \times$ the upper limit of normal [ULN] or calculated creatinine clearance of $\geq 50 \mathrm{~mL} / \mathrm{min}$; bilirubin less than 1.5 $\mathrm{mg} / \mathrm{dL}$; hepatic enzymes $<3$ times the ULN; white blood cell count $\geq 4,000 / \mathrm{mm}^{3}$; and platelet count $\geq 100,000 /$ $\mathrm{mm}^{3}$ ). The patient cohort included cases undergoing upfront surgery, interval cytoreduction after neoadjuvant chemotherapy (NACT), and those undergoing surgery for recurrent disease. Patients with limited peritoneal dissemination (peritoneal cancer index $[\mathrm{PCI}]<7$ ) confirmed by laparoscopy or laparotomy were subjected to CRS/HIPEC. Patients with high disease burden and massive ascites were referred for NACT. Informed consent was obtained from all patients. Ethics committee (EC) approval and Institutional Review Board (IRB) approval was obtained. All patients were treated by a team of two surgeons, anesthesiologist,

Table 1 Perioperative patient characteristics

\begin{tabular}{|l|l|}
\hline Variable & $\boldsymbol{n}$ \\
\hline Age (years) median & 55.5 \\
\hline Hemoglobin $(\mathrm{g} / \mathrm{dl})$ mean & 11.5 \\
\hline Albumin $(\mathrm{g} / \mathrm{dl})$ mean & 3.8 \\
\hline Performance status & \\
\hline ECOG 0 & 12 \\
\hline ECOG 1 & 4 \\
\hline Comorbidity & 4 \\
\hline Prior surgical score & \\
\hline 0 & 16 \\
\hline 1 & 0 \\
\hline 2 & 0 \\
\hline 3 & 0 \\
\hline NACT & \\
\hline Yes & 6 \\
\hline Interval & 10 \\
\hline
\end{tabular}

Abbreviations: ECOG, Eastern Cooperative Oncological Group; NACT, neoadjuvant chemotherapy. intensivist, and medical oncologist having expertise in peritoneal surface malignancy. The extent of intraperitoneal tumor manifestation is determined using the $\mathrm{PCI}$ and a combined numerical score of lesion size (LS-0 to LS-3) and tumor localization (region $0-12$ ). ${ }^{1}$ The aim of CRS is to obtain optimal cytoreduction (defined as CCR-0/1) as a precondition for the application of HIPEC. Following cytoreduction, all patients underwent HIPEC by semiopen technique with a dedicated HIPEC machine (RanD Biotech) using injection mitomycin $35 \mathrm{mg} / \mathrm{m}^{2}$. Patient baseline demographics and perioperative details like $\mathrm{PCI},{ }^{2}$ prior surgical score (PSS), the average blood loss, operative time, hospital stay, and ICU stay were recorded prospectively in all patients. Adverse events are graded according to common terminology criteria for adverse events (CTCAE). ClavienDindo classification was used to grade surgical complications. In-hospital mortality was recorded. Histopathology was assessed by dedicated oncopathologist. The patients were followed up with regular upper gastrointestinal (GI) endoscopy and radiological monitoring for any recurrence.

\section{Statistical Analysis}

Continuous variables are presented as mean with standard error of the mean or median with range or interquartile range (IQR), as appropriate. Adverse events were recorded and graded according to the CTCAE version 4.0. Survival was calculated in a Kaplan-Meier survival curve. Progressionfree survival (PFS) and overall survival (OS) both were calculated from date of surgery.

\section{Results}

A total of 16 patients with gastric cancer and peritoneal carcinomatosis were operated from February 2015 to December 2019 at Manipal Comprehensive Cancer Center. The median age of our patients was 55.5 years. Prior surgical score was 0 for all 16 patients. Six patients underwent upfront CRS, while 10 patients underwent interval CRS after NACT. The mean number of cycles of NACT was 4.

The mean $\mathrm{PCI}$ was 3.5 (range 1-7), and adequate complete cytoreduction (CC) score of 0/1 was achieved in all patients. The mean duration of surgery was 8.5 hours; mean intraoperative blood loss was $575 \mathrm{~mL}$. Total gastrectomy and D2 lymphadenectomy were performed in all patients. Total peritonectomy was performed in two patients; pelvic peritonectomy was performed in 6 patients; and total omentectomy and lesser omentectomy were performed in all patients. Bilateral salpingo-oophorectomy was performed in two patients. Multivisceral resection ( $>4$ organs' resection) was done in one patient. All patients received HIPEC with $35 \mathrm{mg} / \mathrm{m}^{2}$ of mitomycin C.

Seven patients were extubated in OT. Median intensive care stay was 2 days. Major surgical complications were observed in 2 patients (12.5\%) who had major surgical site infections. Grade I surgical site infection was present in two patients. Six patients had prolonged GI recovery. The 30-day mortality was zero. 

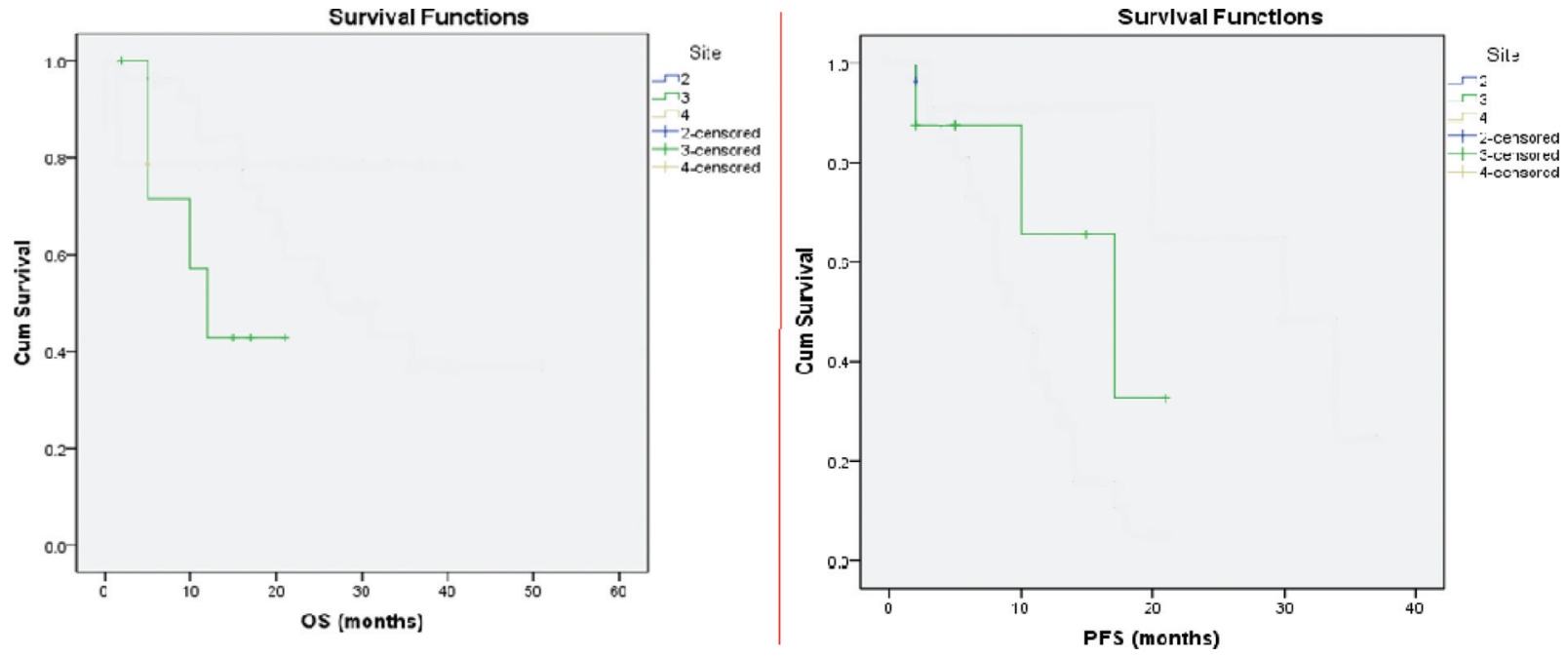

Fig. 1 Overall survival (OS) curve and progression-free survival (PFS) curve.

Median follow-up time was 39 months. The median OS was 12 months (95\% CI 6.86-17.13]. The median PFS was 17 months (95\% CI 6.36-27.64) (-Fig. 1).

\section{Discussion}

Systemic chemotherapy has improved the prognosis of patients with metastatic gastric cancer, reaching survival of 8 to 14 months in selected series. ${ }^{3}$ However, the response rate of measurable gastric peritoneal carcinomatosis to systemic chemotherapy is only 14 to $25 \%$, most likely attributable to poor penetration of systemic chemotherapy across the blood-peritoneal barrier. ${ }^{4}$

A meta-analysis by Yan et $\mathrm{al}^{5}$ on adjuvant regional chemotherapy for resectable gastric cancer has shown a survival benefit from addition of intraperitoneal chemotherapy. A significant improvement in survival was associated with HIPEC (hazard ratio $[\mathrm{HR}]=0.60 ; 95 \% \mathrm{CI}=0.43$ to 0.83 ; $p=0.002)$ or HIPEC combined with early postoperative intraperitoneal chemotherapy ( $\mathrm{HR}=0.45 ; 95 \% \mathrm{CI}=0.29$ to $0.68 ; p=0.0002$ ). They also noted that Intraperitoneal chemotherapy was also found to be associated with higher risks of intra-abdominal abscess ( $R R=2.37 ; 95 \% \mathrm{CI}=1.32$ to 4.26 ; $p=0.003)$ and neutropenia $(\mathrm{RR}=4.33 ; 95 \% \mathrm{CI}=1.49$ to $12.61 ; p=0.007)$.

A recent randomized control trial by Rudloff et $\mathrm{al}^{7}$ comparing CRS-HIPEC with systemic chemotherapy demonstrated an overall survival of 11.3 months in CRS-HIPEC arm versus 4.3 months in the chemotherapy alone arm.

Despite several studies reporting encouraging survival results with CRS + HIPEC in patients with gastric cancer and peritoneal carcinomatosis, ${ }^{3,8-10}$ its use has not been standardized. PCI and CC scores are known to be independent prognostic factors after CRS plus perioperative chemotherapy.

From a systematic review and meta-analysis of data from 748 patients with peritoneal metastasis, Coccolini et al ${ }^{11}$ concluded that the PCI cutoff level can be 12 for better or worse prognosis. Accordingly, peritonectomy and CRS are not recommended in patients with PCI higher than the cut-off level. Yonemura et $\mathrm{al}^{12}$ could show in a multivariate analysis that the completeness of cytoreduction is a highly significant factor for the prediction of patient survival. He also determined that the best results are obtained with $\mathrm{PCI}<6$.

The optimal cytoreduction, measured as CC score showed a survival benefit, according to a systematic review by Coccolini et al. The OS increased by CCO-CC1 cytoreduction in patients with peritoneal carcinomatosis of gastric origin.

Our study results, despite limited number of patients with a mean follow-up of 38 months, show recurrence-free and OS rates comparable with earlier published studies. Mean PCI of our study was 3.5, which is a good prognostic factor, and optimal cytoreduction (CC score 0/1) that we were able to achieve in all our patients may have contributed to this.

Therefore, the use of CRS-HIPEC for selected patients with gastric cancer and peritoneal carcinomatosis seems to improve survival, as evidenced by other systematic reviews. ${ }^{13}$ Although the survival benefit is less encouraging than those obtained for other peritoneal surface malignancies, a multidisciplinary approach for physically fit, low burden disease with complete cytoreduction can be benefited.

\section{Conclusion}

Multidisciplinary treatment of perioperative chemotherapy with CRS and HIPEC is a promising treatment option, which may prolong survival in selected patients (low peritoneal disease burden, physically fit patients) with gastric carcinomatosis. Large randomized clinical trials are warranted to prove its efficacy and become a standard of care.

Funding

The authors declare that they have not received any funding.

\section{Conflict of Interests}

The authors declare that they have no competing interests. 


\section{Acknowledgment}

Dr. Arun Kumar, Consultant Biostatistician, for statistical analysis and sample size calculation.

\section{References}

1 Glehen O, Gilly FN. Quantitative prognostic indicators of peritoneal surface malignancy: carcinomatosis, sarcomatosis, and peritoneal mesothelioma. Surg Oncol Clin N Am 2003;12(03):649-671

2 Jacquet P, Sugarbaker PH. Clinical research methodologies in diagnosis and staging of patients with peritoneal carcinomatosis. Cancer Treat Res 1996;82:359-374

3 Rihuete Caro C, Manzanedo I, Pereira F, Carrion-Alvarez L, Serrano Á, Pérez-Viejo E. Cytoreductive surgery combined with hyperthermic intraperitoneal chemotherapy (HIPEC) in patients with gastric cancer and peritoneal carcinomatosis. Eur J Surg Oncol 2018;44(11):1805-1810

4 Yano M, Shiozaki H, Inoue M, et al. Neoadjuvant chemotherapy followed by salvage surgery: effect on survival of patients with primary noncurative gastric cancer. World J Surg 2002;26(09):1155-1159

5 Yan TD, Black D, Sugarbaker PH, et al. A systematic review and meta-analysis of the randomized controlled trials on adjuvant intraperitoneal chemotherapy for resectable gastric cancer. Ann Surg Oncol 2007;14(10):2702-2713

6 Sugarbaker PH, Cunliffe WJ, Belliveau J, et al. Rationale for integrating early postoperative intraperitoneal chemotherapy into the surgical treatment of gastrointestinal cancer. Semin Oncol 1989;16(4, Suppl 6)83-97
7 Rudloff U, Langan RC, Mullinax JE, et al. Impact of maximal cytoreductive surgery plus regional heated intraperitoneal chemotherapy (HIPEC) on outcome of patients with peritoneal carcinomatosis of gastric origin: results of the GYMSSA trial. J Surg Oncol 2014;110(03):275-284

8 Hultman B, Lind P, Glimelius B, et al. Phase II study of patients with peritoneal carcinomatosis from gastric cancer treated with preoperative systemic chemotherapy followed by peritonectomy and intraperitoneal chemotherapy. Acta Oncol 2013;52 (04):824-830

9 Coccolini F, Cotte E, Glehen O, et al. Intraperitoneal chemotherapy in advanced gastric cancer. Meta-analysis of randomized trials. Eur J Surg Oncol 2014;40(01):12-26

10 Desiderio J, Chao J, Melstrom L, et al. The 30-year experience-A meta-analysis of randomised and high-quality non-randomised studies of hyperthermic intraperitoneal chemotherapy in the treatment of gastric cancer. Eur J Cancer 2017;79:1-14

11 Coccolini F, Catena F, Glehen O, et al. Complete versus incomplete cytoreduction in peritoneal carcinosis from gastric cancer, with consideration to PCI cut-off. Systematic review and meta-analysis. Eur J Surg Oncol 2015;41(07):911-919

12 Yonemura Y, Bandou E, Kawamura T, Endou Y, Sasaki T. Quantitative prognostic indicators of peritoneal dissemination of gastric cancer. Eur J Surg Oncol 2006;32(06):602-606

13 Ellison LM, Man Y, Stojadinovic A, Xin H, Avital I. Cytoreductive surgery and hyperthermic intraperitoneal chemotherapy in treatment of gastric cancer with peritoneal carcinomatosis. Chin J Cancer Res 2017;29(01):86-92 